\title{
Research Technologies of Projected Capacitive Touch Screen
}

Xiyang Liu ${ }^{a}$, Siling Feng ${ }^{b}$,Xianmei Chen ${ }^{c}$,Jinrong Liu ${ }^{d}$,Yaochi Zhao ${ }^{\mathrm{e}}$

School of College of Information Science and Technology, Hainan university, Hainan Haikou 570228 , China

aliuxiyang0719@163.com, lily85161@163.com, ${ }^{\mathrm{c}} 79901 @ 163 . c o m$,

dLiujinrong98@163.com, ${ }^{\mathrm{e} y a o c h i z i @ 163 . c o m ~}$

Keywords: Projected capacitive touch screen, ITO, Gravity method, SNR, Bilinear Interpolation.

Abstract. Projected capacitive touch screen has the advantage of wear-resistant, high transmittance, long life, low power consumption, high sensitivity, noise rejection capability and support for multi-touch .This paper studies the related technologies of projected capacitive touch screen system: full touch point detection technology of mutual capacitance, gravity method to locate the touch point of the finger, IIR digital filter to improve the signal to noise ratio, bilinear interpolation method to achieve the rotation and scaling of the image.

\section{Introduction}

Touch screen has been widely used in mobile phones, PAD and computers. Touch screen can be divided into resistive, capacitive, infrared and surface-acoustic wave based on the induction principle[1]. Capacitive touch screen has the advantage of wear-resistant, long life, low power consumption, high sensitivity, noise rejection capability and support for multi-touch . When a finger or other conductor is near the projected capacitive touch screen, electric field at the touching point will be affected, resulting in increasing in the self-capacitance and reducing in the mutual capacitance at touch point.

The touch screen is three-layer structure: the middle layer is an insulating layer, the upper and lower layers are indium tin oxide (ITO) layer. The two ITO layers of the capacitive touch screen insulated by the insulation layer are respectively made by $\mathrm{M}$ rows and $\mathrm{N}$ columns diamond induction embedded in mutual arrangement, ITO diamond-ground capacitance of a row or a column is self-capacitor, ITO diamond capacitance between a row and a column intersect block is mutual capacitance. Structure of the projected capacitive touch screen is shown in Figure 1.

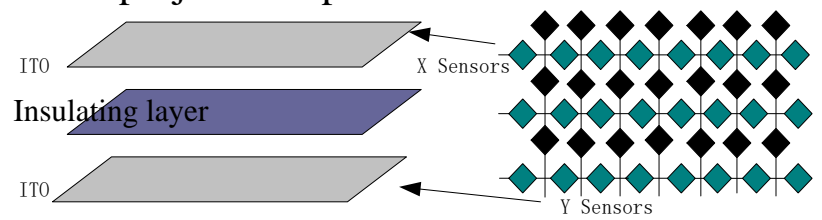

Fig.1 Structure of the projected capacitive touch screen

Visible, a plurality of sensing electrodes on the sensing area of projected capacitive touch screen are arranged respectively $\mathrm{X}, \mathrm{Y}$ direction and are crossing each other, sensing electrode size is about $1 \mathrm{~mm}$. For mutual capacitance, when a finger approaches or touches the touch screen, as a human finger is equivalent of conductor, a part of electric field or power-line between the induction block and the receiver block is transferred to the finger, the field or power lines between the two sensing blocks reduced, equivalent mutual capacitance reduction[2].

Therefore, it is necessary to detect efficiently, rapidly and accurately the capacitance changing value of the sensing electrodes-ground, which reflects the touch action about finger on the touch screen, so the related technologies of projected capacitive touch screen include full contact detection technology, finger location, improvement signal to noise ratio, realization of image rotation and scaling, etc. 


\section{Full-contact detection technology for mutual capacitance}

Mutual capacitance value of the touch sensing module may be obtained by cross-scanning. Count value of each sensor is obtained by cross-scanning within an period of time, and through the software whether there is a finger touch is determined by the change of the count value. Due to scan all intersections on the entire screen, cross point number of scans the entire screen is implemented once a full scan of $\mathrm{M} * \mathrm{~N}$ times[3].

Each intersection block of rows and columns themselves are self-capacitance. There is mutual capacitance between rows and columns. Each sensing module also has a block resistance. The self-capacitance of an unit block of rows and columns are, respectively $\mathrm{Cr}$ and Cc. The mutual capacitance between the rows and columns is expressed as Crc. The sensing resistor block are represented by $\mathrm{Rr}$ and $\mathrm{Rc}$ of rows and columns. The unit block model and circuit model are shown in Figure2.
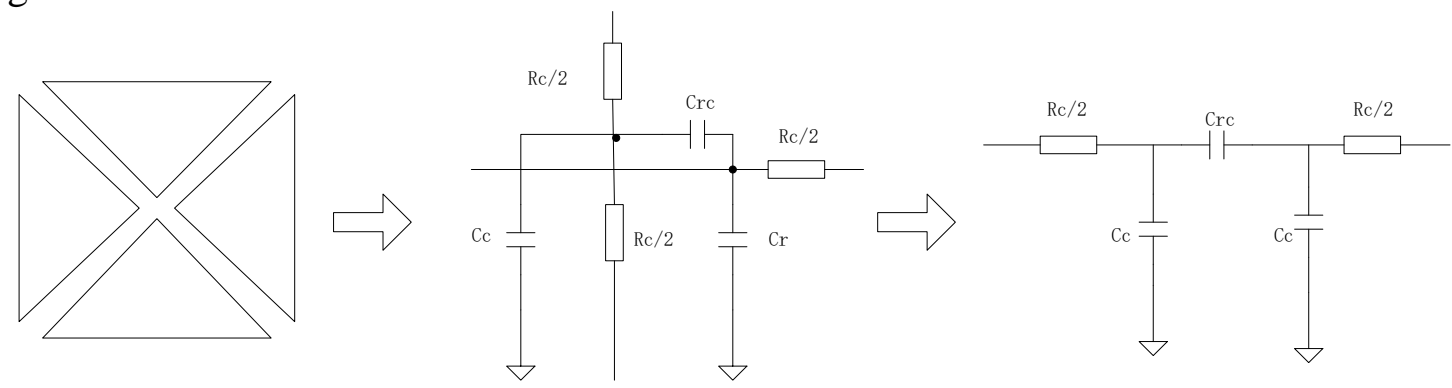

Fig.2 Unit block model and circuit model

A high frequency square wave excitation signal is sent to the line $\mathrm{X}$ screen driven by the PSoC chip. Since the mutual capacitance exists, the screen may receive the same frequency signal as the transmitted signal at the column Y. Mutual capacitance at the intersection of row and column Cm is approximately equal to Crc. Self-capacitance of rows and columns are no longer $\mathrm{Cr}$ and $\mathrm{Cc}$, but the equivalent self-capacitance Csr and Csc; Resistance of rows and columns is no longer Rr/2 and $\mathrm{Rc} / 2$, but from the emission of the line to the intersection line resistance Rsr and from receiving endpoint to the intersection column resistance Rsc.

The column $\mathrm{Y}$ receives the signal as same frequency as the transmitted signal from the line $\mathrm{X}$, at the same time, there is an alternating current from row Tx to column Rx. The size of the alternating current is directly related to the mutual capacitance $\mathrm{Cm}$. The greater $\mathrm{Cm}$ is, the greater current is, on the contrary, the smaller the current is. The alternating current into direct current through the rectifier/filter. The direct current is converted into a direct voltage through the I/U conversion. The direct voltage is converted into a digital signal by the A/D conversion . The data processing converters the change in the value of $\mathrm{Cm}$ into the change the of the system clock period. Acquisition principle diagram of mutual capacitance $\mathrm{Cm}$ is shown in Figure 3.

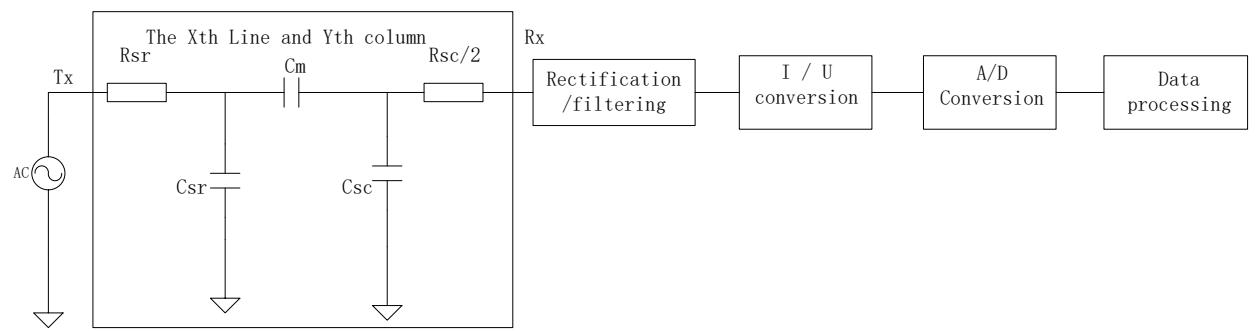

Fig.3 Acquisition principle diagram of mutual capacitance $\mathrm{Cm}$

When a finger touches the touch screen, Cm reduces and self-capacitance Csr and Csc increased. Reducing of mutual capacitance and increasing of self-capacitance result in decreases in the current from row Rx to column Tx, and ultimately the value of A/D conversion is reduced. The software in PSoC chip controls cross point scanning circuit and makes it scan all cross points on the screen and monitor each intersection of $A / D$ conversion values. If it exceeds a preset threshold value, there is considered to have a finger touch. The signal size of the intersection, combined with the size of the adjacent intersection signal changes to a finger touch position to pinpoint.

When there is more than one finger touch on the touch-screen surface, PsoC chip issues 
time-scanning pulse in the driving electrodes ( $\mathrm{X}$ direction). When applying a high frequency signal in a horizontal drive electrodes, due to the presence of coupling capacitors, sensing high-frequency signal can be detected in sensing electrode in the longitudinal direction (Y direction), signal strength is closely related to the size of the coupling capacitance[4]. When there are the multi-point (usually no more than five points) touch simultaneously, the sensing electrodes receive multi-points signals change. Because transmission and reception are sharing proceed, it can eliminate the "ghost".

\section{Positioning finger}

User location on the touch screen typically requires less than $1 \mathrm{~mm}$, Due to the diamond-shaped pieces size of the touch screen is designed on the diagonal, usually $4 \sim 6 \mathrm{~mm}$, so when a finger touching, generally there are four diamond-shaped pieces touched (horizontal and vertical each 2), even there may be more. According to the different signal amplitude of the finger touch the row and column, the procedure from locating the center of gravity method was used. Positioning finger on the touch screen is shown in Figure 4.

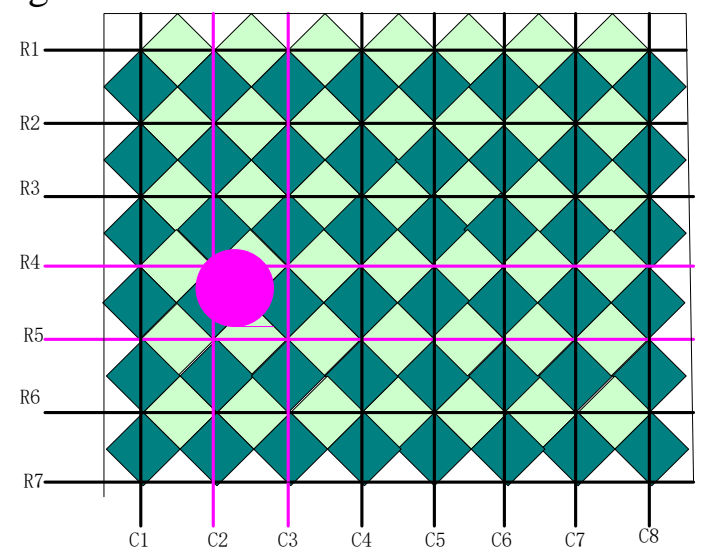

Fig 4. Positioning finger on the touch screen

In order to meet the accuracy requirements, the modified center of gravity is as formula (1).

$$
P=\frac{R_{L}}{N} \times \frac{\sum_{n=1}^{N} n \bullet S_{n}}{\sum_{n=1}^{N} S_{n}}-0.5
$$

Where $\mathrm{P}$ is the coordinate value of the finger on the screen of the $\mathrm{X}$ or $\mathrm{Y}$; $\mathrm{G}$ is the amplification factor; $S_{n}$ is the signal values of the n-th diamond block; $\mathrm{N}$ is the number of the $\mathrm{X}$ or $\mathrm{Y}$ direction diamond block; $R_{L}$ is the logical resolution of touch screen on the $\mathrm{X}$ or Y direction.

\section{Digital filtering to improve SNR}

SNR is the ratio of finger touch signals measured on the sensor block and noise induced on a block of the touch screen. Signal sensor acquired often mixed with noise and various interference signals, Moreover, the different scanning frequencies, proximal or distal finger signal differences will affect the sensitivity of the touch screen, that will affect the signal to noise ratio [5]. To improve the signal to noise ratio, an infinite impulse response (IIR) filter is used to filter effectively the noise of scanning sample values.

IIR digital filter is a kind of recursive causal linear time-invariant systems, its current output value is determined jointly by the current and historic input value and the historic output value, and in order to ensure the stability of the filter, the system function poles of IIR digital filter should be located in the unit circle of the $\mathrm{z}$ plane. The feature of a first-order IIR digital filter is that its output $\mathrm{y}$ (n) is determined commonly by the current input signal $\mathrm{x}$ (n) and past output signal $\mathrm{y}$ (n-1) .decision. A first-order IIR filter is shown in Figure 5. 


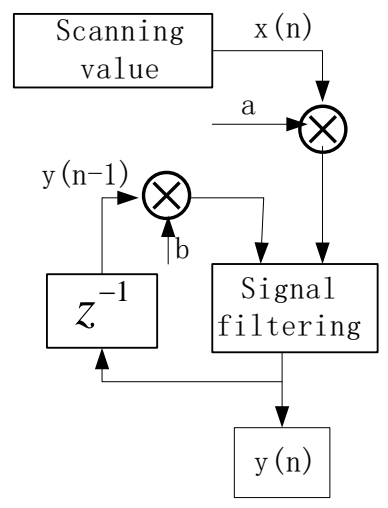

Fig 5. IIR filter

The differential equation of input and output is as equation (2).

$y(n)=a \times X(n)+b \times y(n-1)$

The corresponding system functions is following formula (3).

$$
H(z)=\frac{Y(z)}{X(z)}=\frac{a}{1-b z^{-1}}
$$

Where the constants $a$ and $b$ must satisfy $b=1-a$. When taking the parameters $a=b=1 / 2$, on the 3.5-inch touch screen experiment, jitter noise has been obviously reduced, SNR has been obviously improved.

\section{To realize the image rotation and scaling}

There is an image rotation and scaling on touch screen, and to minimize the distortion of the image, bilinear interpolation algorithm for image rotation and scaling was used. Bilinear interpolation is linear interpolation extension of two variables function, the core idea is a respective linear interpolation in two directions[6].

In image bilinear interpolation algorithm, the newly creating pixel values of the target image is obtained through a weighted average calculation by four neighboring pixel values in $2 * 2$ area of the source image. For a target pixel, setting the coordinates is to reverse transform ,the floating point coordinates is $(i+u, j+v)$, (Where $\mathrm{i}$ and $\mathrm{j}$ are the integer part of floating point coordinates, $\mathrm{u}$ and $\mathrm{v}$ are the fractional part of the floating-point coordinates, the value of $[0,1)$ floats), Then the pixel values $f(i+u, j+v)$ is decided by the surrounding four pixels value, their coordinates in source image is $(i, j) 、(i+1, j) 、(i, j+1)$ and $(i+1, j+1)$, as following formula (4).

$$
f(i+u, j+v)=(1-u)(1-v) f(i, j)+(1-u) v f(i, j+1)+u(1-v) f(i+1, j)+u v f(i+1, j+1)
$$

Where $f(i, j)$ represents the pixel value of the source image, and so on.

Bilinear interpolation algorithm makes full use of the four real pixel values around the virtual point in source image, which together determine a target image pixel value, discontinuity of pixel values do not happen, it is good zoom effect. The original image is shown in Figure 6, the rotation and scaling image is shown in Figure 7. 


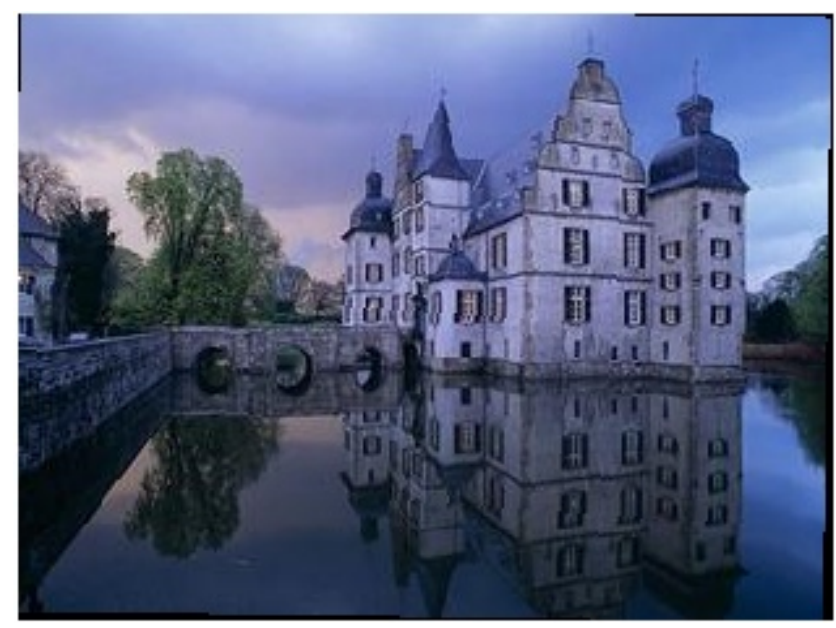

Fig 6. The original image

\section{Conclusions}

The above technologies for small-sized projected capacitive touch screen detection system, the original data processing meets the requirements of the touch screen, namely the acquisition of the signal processing is right, capacitance data adjusting accuracy is $95.3 \%$.

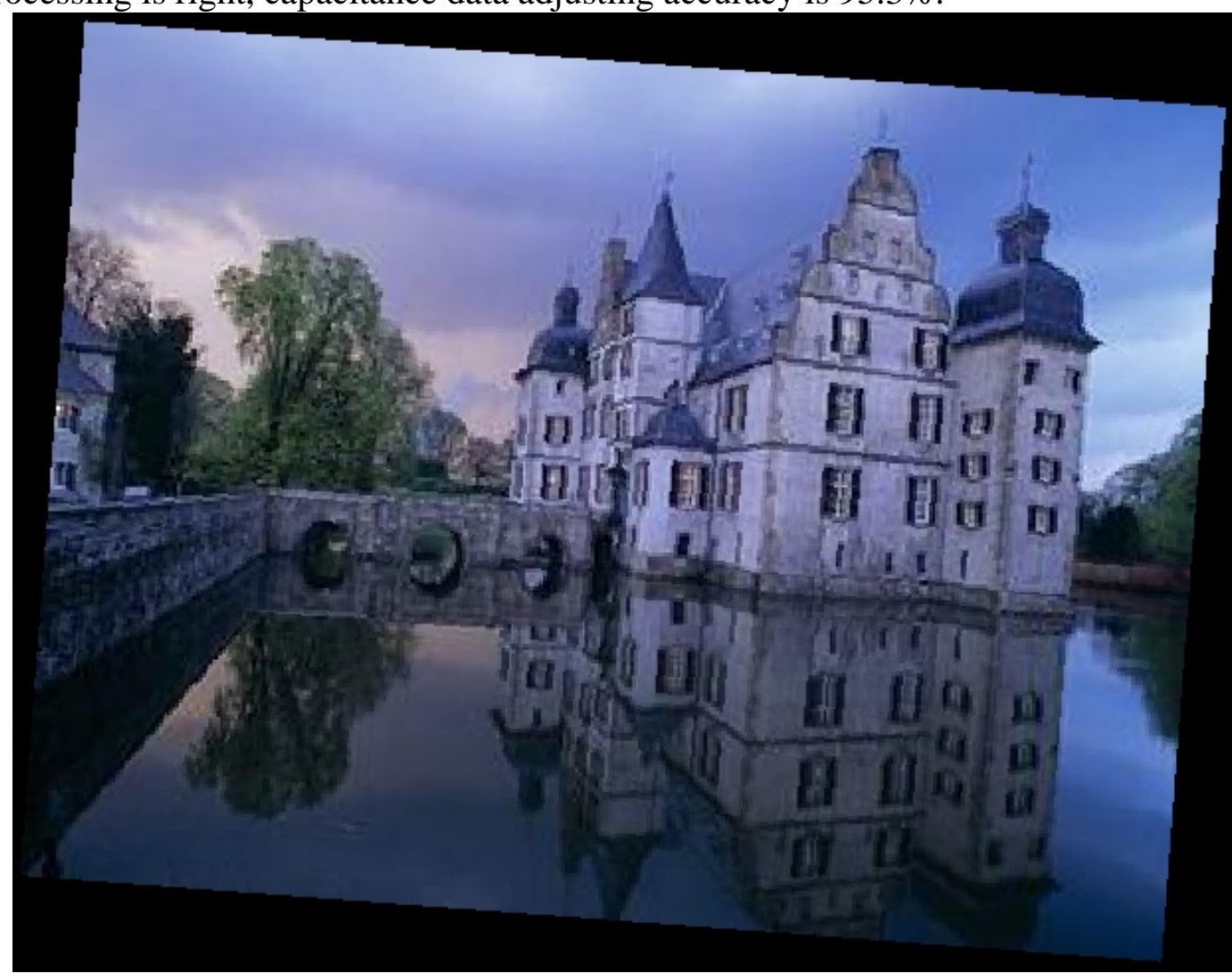

Fig 7. The rotation and scaling image

\section{Fund Project}

1.Hainan social development science and technology projects: Research of the human plantar pressure characteristics Identification based on fuzzy theory(Item Number: 2015SF33) .

2.Hainan Natural Science Fund: Biogeography Optimization Algorithm Based on Multi-objective to Solve Biological Motif and Discovery Problem(Item Number: 20156226) .

3.Hainan Natural Science Fund: Key Technology Research of Smart Video Object Segmentation and Tracking Based on DRLSE(Item Number: 20156228). 


\section{References}

[1].KNOWLES TJ. Touch panel for an acoustic touch position sensor[p]. US Patent:5,329,070,1994-07-12.

[2].Yongsuk Park. Maximizing Responsiveness of Touch Sensing via Charge Multiplexing in Touch Screen Devices[J].IEEE Transactions on Consumer Electrons, Vol.56,No.3,August 2010:1905-1910.

[3].Harley J A, Orsley T J. Single Layer Mutual Capacitance Sensing Systems, Device, Components and Methods: US, US20090194344 A1[P]. 2009.

[4]. Hotelling S P. Mutual capacitance touch sensing device: US, US8514185 B2[P]. 2013.

[5].Tong-Hun Hwang. A Highly Area-Efficient Controller for Capacitive Touch Screen Panel Systems[J].IEEE Transactions on Consumer Electrons,Vol.56,2,May 2010:1115-1121

[6].Lu J, Xiong S, Wu S. An Improved Bilinear Interpolation Algorithm of Converting Standard-definition Television Images to High-definition Television Images[C]// 2009. 2009:441-444. 